\title{
Exogenous temporal cues enhance recognition memory in an object-based manner
}

\author{
JUNJI OHYAMA \\ University of Tokyo, Tokyo, Japan \\ AND \\ KaTSUMi Watanabe \\ University of Tokyo, Tokyo, Japan \\ National Institute of Advanced Industrial Science and Technology, Tokyo, Japan \\ and Japan Science and Technology Agency, Saitama, Japan
}

\begin{abstract}
Exogenous attention enhances the perception of attended items in both a space-based and an object-based manner. Exogenous attention also improves recognition memory for attended items in the space-based mode. However, it has not been examined whether object-based exogenous attention enhances recognition memory. To address this issue, we examined whether a sudden visual change in a task-irrelevant stimulus (an exogenous cue) would affect participants' recognition memory for items that were serially presented around a cued time. The results showed that recognition accuracy for an item was strongly enhanced when the visual cue occurred at the same location and time as the item (Experiments 1 and 2). The memory enhancement effect occurred when the exogenous visual cue and an item belonged to the same object (Experiments 3 and 4) and even when the cue was counterpredictive of the timing of an item to be asked about (Experiment 5). The present study suggests that an exogenous temporal cue automatically enhances the recognition accuracy for an item that is presented at close temporal proximity to the cue and that recognition memory enhancement occurs in an object-based manner.
\end{abstract}

The world around us changes continuously, but our memories of the world are fragmentary. The memory system may often become overloaded and fail because of a vast amount of new and changing information. Thus, we resort to recalling fragmentary scenes that are abstracted from important parts of ever-changing data streams. For instance, it is likely that an individual will be unable to report the totality of his or her experience of a previous day but will, instead, be able to recall a few salient or interesting scenes.

What determines which item is memorized? There has been substantial interest in the role of an item's characteristic in memory priority. In several studies, multiple items were presented simultaneously for short durations. If one of them had some unique characteristic and the others did not, participants' immediate recall of the former was better. The distinguishing characteristics, which increased the priority in memory, may be conferred by an item's saliency, familiarity, or positivity (Fine \& Minnery, 2009; Jackson \& Raymond, 2008; Jackson, Wu, Linden, \& Raymond, 2009). Since the participants could freely choose the order in which to attend to multiple items in these studies, the reports explained that the order of attention or memory input presumably was related to an item's characteristics. The participants might have paid attention to and memorized the simultaneously presented items in the attended order until they reached the limit of their memory capacities (Baddeley, 2003; Cowan, 2001; Kintsch, Patel, \& Ericsson, 1999).

Salient, peculiar, and interesting stimuli can automatically attract attention (e.g., Posner, 1980; Prinzmetal, Presti, \& Posner, 1986), a process that enhances the sensory processes (detection and discrimination) of attended stimuli (e.g., Desimone \& Duncan, 1995; Jonides, 1981; Yantis, 1996; Yeshurun \& Carrasco, 1998, 1999). Likewise, focusing attention on a spatial location, whether voluntarily or involuntarily, increases the probability that information at that location will be transferred into visual working memory (e.g., Bundesen, 1990; Duncan \& Humphreys, 1989; Luck \& Thomas, 1999; Schmidt, Vogel, Woodman, \& Luck, 2002; Wolfe, Horowitz, \& Michod, 2007), resulting in better recognition or recall of the attended stimuli.

Visual attention studies have suggested two modes of attention - space based and object based. In the former, stimuli are selected by a location in the visual field (Posner, 1980). In spatial precuing tasks, an abrupt change attracts attention to the location. As mentioned above, space-based attention has been shown to lead to enhanced memory for visual stimuli at the attended location (e.g., Schmidt et al., 2002; Wolfe et al., 2007). In contrast, attention can also be directed to an object or a coherent form 
in the environment. For instance, Egly, Driver, and Rafal (1994) demonstrated that detecting a target at a cued location was the quickest way but that detecting targets at the uncued location of a cued object was faster than detecting those at the location of an uncued object. This result indicates that the cue directed attention to the entire cued object. Many studies have confirmed this object-based attentional effect on sensory processes (Baylis \& Driver, 1992; Behrmann, Zemel, \& Mozer, 1998; Kramer \& Jacobson, 1991; Lavie \& Driver, 1996; Moore, Yantis, \& Vaughan, 1998; Müller \& Kleinschmidt, 2003; Shomstein \& Behrmann, 2008; Watson \& Kramer, 1999). However, no study has examined whether object-based exogenous cuing enhances recognition memory.

In the present study, we examined whether memory enhancement by task-irrelevant visual changes would occur in an object-based manner. Participants were required to memorize serially presented multiple letters and were asked to recognize one of the letters (target letter) following the perception of the letter sequence. A sudden visual change in a task-irrelevant background stimulus occurred independently, with the letter sequence as an exogenous cue. The presentation rate of the letter sequence was set to be relatively low in order to allow robust encoding of individual letters (see, e.g., Bundesen \& Harms, 1999; Potter, Staub, Rado, \& O'Connor, 2002). In Experiment 1, the exogenous cue occurred at the same location as the location of the letter sequence. In Experiment 2, the cue was presented on the opposite side of the visual field (i.e., different location). We hypothesized that the exogenous cue would enhance recognition performance for a letter only when the cue and the letter were presented at the same location and time. The subsequent experiments (Experiments 3 and 4) were designed to determine whether memory enhancement by the exogenous cue is strictly location based or whether it can be object based. Finally, Experiment 5 examined whether the enhancement of recognition memory would occur even when the exogenous cue was counterpredictive of the timing of the letter to be asked about (target).

\section{EXPERIMENT 1}

In Experiment 1, we examined the effect of a sudden visual change of a task-irrelevant stimulus on participants' ability to remember sequentially presented letters; we denote this task as a recognition memory task. In particular, we compared recognition accuracy when the visual cue occurred with accuracy in a control condition in which no change occurred.

Previous literature has reported that attention is automatically and rapidly reoriented to a sudden and/or salient event (Jonides \& Yantis, 1988; Posner, 1980) and that items co-occurring with an event are more accurately recognized (Bundesen, 1990; Duncan \& Humphreys, 1989). Therefore, detection performance for letters in sequence was also examined in a separate session by measuring the time it took to identify one letter that had previously been specified (target letter) and was embedded in a series of distractor letters; we will call this a detection task.

\section{Method}

Participants. Seven naive participants who had normal or corrected-to-normal vision participated in the experiment. They were all engaged in both the recognition memory and detection tasks.

Stimuli and Apparatus. Visual stimuli were presented on a 22in. CRT monitor (100-Hz frame rate). The stimuli consisted of a fixation cross, a series of sequentially presented letters, and two object figures (see Figure 1). The white fixation cross (about $0.8^{\circ}$ in visual angle) was presented at the center of the monitor. The letter sequence consisted of a series of 11 white Roman letters (about $1.0^{\circ}$ in visual angle), located $1.875^{\circ}$ to the left or right of the fixation cross. The object figures were two black disks $\left(1.5^{\circ}\right)$ behind the letter sequence, which were presented $1.875^{\circ}$ to the left and right of the fixation cross. Thus, the letter sequence always appeared at either side of the two black disks.

Figure 1 shows the time course of the stimulus sequence. Each trial was initiated by a participant's buttonpress. The two black disks and fixation cross were presented throughout a trial $(3,780 \mathrm{msec})$. Prior to the onset of the letter sequence, a "+" symbol appeared in one of the two (left or right side) black disks and indicated the position where the letter sequence would appear. The presentation side of the letter sequence was manipulated in the blocked design; it alternated after every 12 trials. Following the pressing of the start

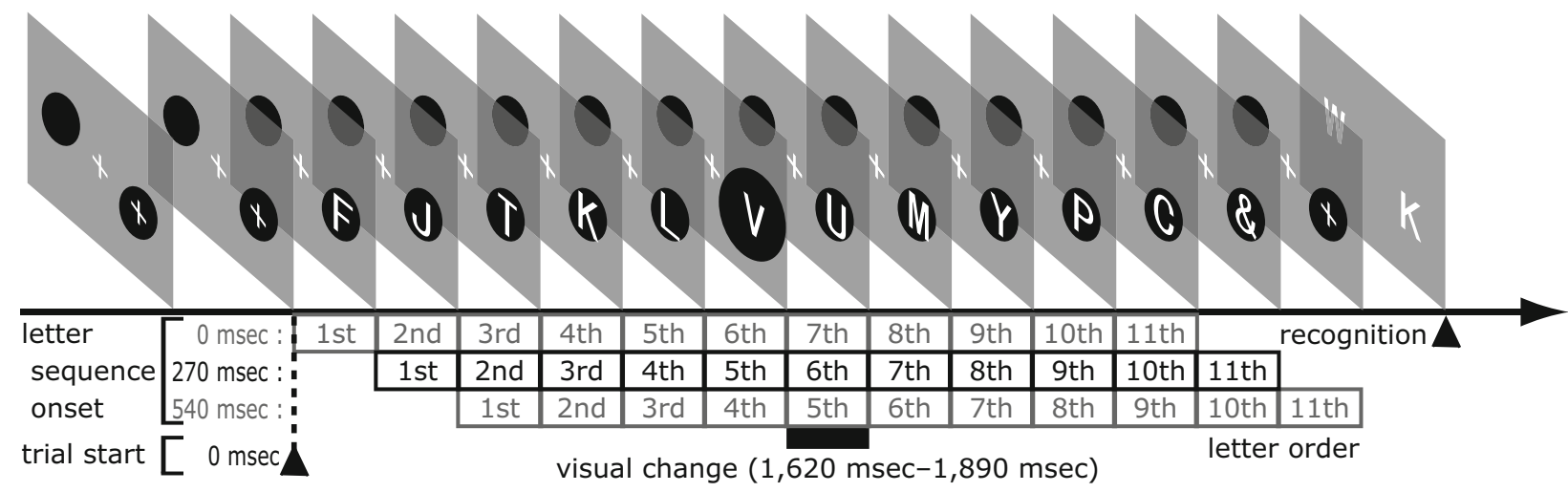

Figure 1. Schematic illustration of stimulus sequence of the recognition memory task in Experiment 1. Participants fixated on the white fixation cross and observed the stimulus sequence. In the change condition, a disk on the same side as the letter sequence was enlarged for the interval from 1,620 to $1,890 \mathrm{msec}$ from the start of the trial, simultaneously with either the fifth, sixth, or seventh letter (i.e., in the figure, the onset time of the letter sequence is $\mathbf{2 7 0} \mathbf{~ m s e c}$ after a trial start; then the visual change occurs simultaneously with the sixth letter). In the no-change condition, the two disks did not change. 
button, the letter sequence appeared at the "+" symbol position. The onset time of the letter sequence from the beginning of a trial was varied $(0,270$, or $540 \mathrm{msec})$. Eleven letters, randomly selected from a set of 20 capital letters (A, B, C, D, E, F, G, H, J, K, L, M, N, P, R, $\mathrm{S}, \mathrm{T}, \mathrm{U}, \mathrm{V}, \mathrm{W})$, appeared sequentially. Following each letter sequence, the "\&" was presented as a mask stimulus. Each letter appeared for $270 \mathrm{msec}$ (3.7 letters per second), with no interstimulus interval, so that the total duration of the letter sequence was 3,240 msec. After the letter sequence, the "+" sign was presented until the trial duration reached $3,780 \mathrm{msec}$

The black disk on the opposite side of the letter sequence never changed throughout the trial. On half of the trials, the size of another black disk (on the same side of the letter sequence) was increased by $50 \%\left(2.25^{\circ}\right)$ for $270 \mathrm{msec}$ (change condition). In the other half, no change occurred (no-change condition). The visual change always occurred at the midpoint in time during each trial (i.e., from 1,620 to $1,890 \mathrm{msec}$ from the start of the trial). The time of the change was fixed. This procedure was to compare the time course of the perception and memory effects by aligning the change condition data and the no-change condition data at the timing of the visual change (or expected timing, in the case of the no-change condition). Moreover, because the onset time of a letter sequence was either 0,270 , or $540 \mathrm{msec}$ from the beginning of the trial, the visual change occurred simultaneously with a presentation of either the seventh, sixth, or fifth letter. Therefore, although the timing of the visual change was predictable in principle, its temporal position in the letter sequence was variable. The order of trials containing change versus no-change conditions was randomized throughout the experiment. Note that although the visual change always occurred at a fixed time from the beginning of the trial, it was difficult to predict at which letter position the visual change would occur, and it was unpredictable whether the visual change would occur. ${ }^{1}$

Recognition memory task. The participants were seated $57 \mathrm{~cm}$ away from the monitor in a dim room. After a variable interval $(0$, 270 , or $540 \mathrm{msec}$ ) from the time of pressing the start button, 11 letters were presented one by one. The participants were instructed to remember all the 11 letters. Immediately after the letter sequence, 2 letters were presented 1.875 visual degrees to the left and right of the fixation cross (see Figure 1). One of them was from the letter sequence that the participants were asked to remember and served as a target letter. The other letter was a letter chosen from the 9 letters that were not in the preceding letter sequence. The participants were asked to report which letter was presented in the preceding sequence by pressing the left or right key on the computer keyboard. Therefore, only one letter was tested on each trial for recognition, although the participants had to memorize as many of the 11 letters in the sequence as possible.

We also varied the temporal position of the to-be-asked-about (target) letter in the letter sequence, relative to the visual change; the target letter appeared at $-2,-1,0,+1$, or +2 items of the letter sequence, relative to the visual change. For each combination of change condition (two: change and no-change) and temporal position (five: $-2,-1,0,+1$, and +2 relative timings of the visual change), 36 trials were repeated (360 trials). In addition, catch trials were incorporated; 6 trials for each of the other six peripheral temporal positions were randomly inserted to eliminate the possible use of memorization of just the five letters at the temporal positions of interest around the visual change time ( 72 trials). Thus, there was a total of 432 trials.

Detection task. The presentation of the visual stimulus was almost identical to that in the recognition memory task. However, in the detection task, the participants were given a target letter prior to the beginning of the trial. The preceding letter was 1 of the 11 letters following the presentation of the serial letter sequence, which also served as a target to be detected. The participants were instructed to distinguish the previously specified target letter from all the other nontarget letters (i.e., distractor letters). They were told to press an appropriate key as quickly as possible once they detected the target letter. The response time (RT) for target detection was recorded. They were not asked to remember the letters in the sequence in this task. Twenty-four trials were repeated for each of the five temporal positions of letter order around the visual change and for each change condition (change vs. no-change [2] $\times$ temporal positions [5] $\times 24$ repetitions $=240$ trials). The other six temporal positions in the letter sequence were randomly queried four times (change vs. nochange [2] $\times$ temporal positions [6] $\times 4$ repetitions $=48$ trials). In total, 288 trials were completed.

\section{Results and Discussion}

With regard to the recognition memory task, Figure 2A depicts recognition memory performance as a function of the temporal position of the letter (i.e., the letter order) relative to the timing of the visual change (that is, from 1,620 to $1,890 \mathrm{msec}$ ), averaged over the participants. Zero on the abscissa reflects the time of the visual change (in the change condition) or the time at which the visual change expected to occur (in the no-change condition). Since target recognition was based on a response to one of two letters, the chance level was $50 \%$. The results indicated that in the no-change condition, recognition performance was about $60 \%$, without much variation among different temporal positions. However, in the change condition, we found a conspicuous peak in the accuracy of a letter recognition that was simultaneously presented with the visual change. In this case, approximately $80 \%$ of the responses were correct, indicating that the participants were much better at recognizing a letter that happened to coincide with the task-irrelevant visual change. A repeated measures ANOVA supported this observation. The main effects both of change condition $[F(1,6)=10.55$, $p<.05]$ and of relative temporal position $[F(4,24)=$ $3.52, p<.05$ ] were significant, as was the interaction $[F(4,24)=3.72, p<.05]$. Post hoc Tukey's HSD tests indicated that the significant main effects and interaction were due to the significant increase in the recognition performance for letters coinciding with the visual change.

The results of the detection task are shown in Figure 2B. The mean RTs (averaged over the participants) for correct detections are plotted as a function of the relative temporal position of a target letter (i.e., a target letter order) with respect to the visual change. RTs less than $200 \mathrm{msec}$ or more than $810 \mathrm{msec}$ (the offset time of the third letter from the target letter) were excluded from the analysis. The mean error rate was $4 \%$. RTs in both of the change conditions became shorter as the target letters appeared later in the sequence, as reflected by the significant main effect of relative temporal position $[F(4,24)=10.47, p<.01]$. This fact probably reflects the foreperiod effect, whereby simple reaction times decrease as the time before the appearance of a target increases (see Niemi \& Näätänen, 1981). However, neither the change condition (change vs. no-change) nor the interaction had much effect on the performance $[F(4,24)=0.52, p=.72$, and $F(1,6)=3.56$, $p=.11$, respectively].

Recognition memory for the item that co-occurred with the task-irrelevant change was higher. This result was consistent with previous findings that spatial atten- 


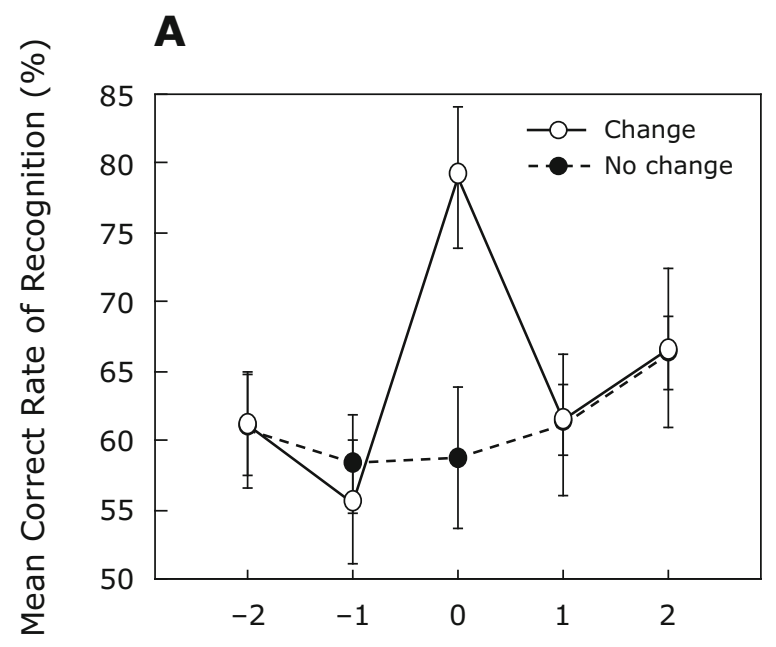

Temporal Position Relative to the Visual Change
B

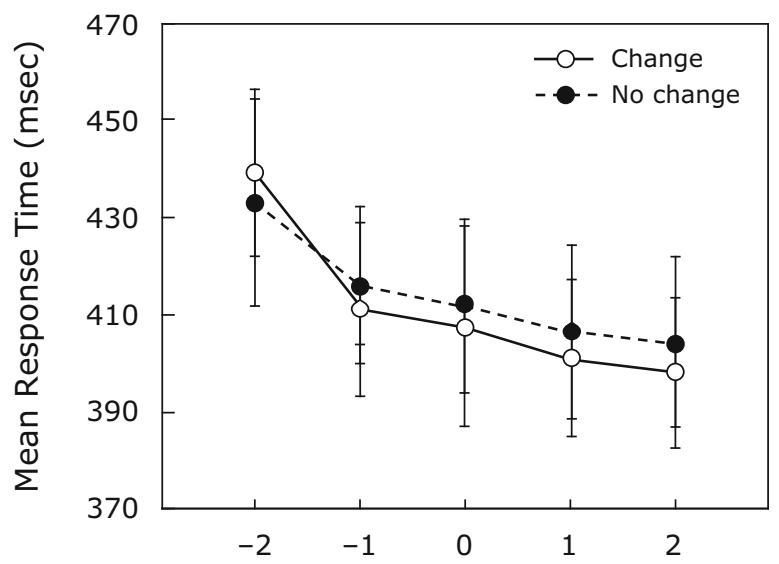

Temporal Position Relative to the Visual Change

Figure 2. (A) Recognition memory accuracy as a function of temporal position of a letter in sequence relative to the timing of the visual change in Experiment 1, averaged over participants. The zero on the horizontal axis corresponds to the fifth, sixth, or seventh letter, depending on the onset time of the letter sequence. (B) Response times for the detection task in Experiment 1, as a function of temporal position of the target letter relative to the timing of the visual change, averaged over participants. Error bars denote the standard errors of the means.

tion enhances the memory formation for visual stimuli at an attended location (e.g., Schmidt et al., 2002; Wolfe et al., 2007). Unexpectedly, we found that target detection time in the detection task was unaffected by the sudden visual change of the task-irrelevant object in the present experiment.

\section{EXPERIMENT 2}

The results of Experiment 1 confirmed that transient visual change enhances recognition memory for visual items occurring at the same time as a change. However, the memory enhancement may simply have been caused by an elevated level of arousal or vigilance evoked by the visual change. If so, the enhancement would not be specific to the location of the visual change. In order to examine the account, we altered the location of the visual change in Experiment 2; it occurred on a disk opposite (contralateral side) to the location of the letter sequence.

\section{Method}

Participants. Seven new participants, all with normal or corrected-to-normal vision, were recruited for Experiment 2.

Stimuli and Procedure. The visual stimuli and experimental procedure were identical to those in Experiment 1, except that the visual change (increase in size of the black disk) occurred at the location (contralateral side) opposite to the letter sequence.

\section{Results and Discussion}

Figure $3 \mathrm{~A}$ presents, in the same format as Figure 2A, the results for the recognition memory task in Experiment 2. It is clear that recognition performance did not differ as a function of either change condition or relative temporal position [repeated measures ANOVA: change condition, $F(1,6)<0.01, p>.99$; temporal position, $F(4,24)=0.86, p=.50$; interaction, $F(4,24)=1.21$, $p=.33]$. Thus, the visual change had little impact on the recognition memory for a simultaneously occurring letter when it was not in close spatial proximity to the letter. The performance in the detection task (Figure $3 \mathrm{~B}$ ) also was not influenced by the visual change $[F(1,6)=3.75, p=.10]$, whereas the RTs for target letter detection were significantly shorter for letters occurring later in the sequences $[F(4,24)=7.95, p<.01]$. There was no significant interaction $[F(4,24)=0.51, p=.73]$.

The results of Experiments 1 and 2, taken together, suggest that memory for a letter is enhanced when a visual change occurs at the same place in space and time as the letter. The detection times for letters in the detection task were unaffected by the sudden visual change in Experiments 1 and 2. The absence of RT differences did not necessarily prove the absence of attentional capture; there was still a possibility that the memory enhancement was caused by the attentional process. The detection of the letter in sequence might be too easy such that the visual change could not disturb or accelerate the RT. However, these findings suggested that visual change effectively modulated the memory process for the recognition of serially presented letters in the same space and time (measured by the recognition memory task), in spite of the fact that the perceptual process for letter detection was easy enough for all the letters in the sequence (measured by the detection task).

In attentional blink (e.g., Folk, Leber, \& Egeth, 2002), a strong misperception of an item is achieved when a distractor cue occurs two frames (or some time) before the onset of the item. However, in our present results, the inhibition of item detection or memory for a subsequent 
A

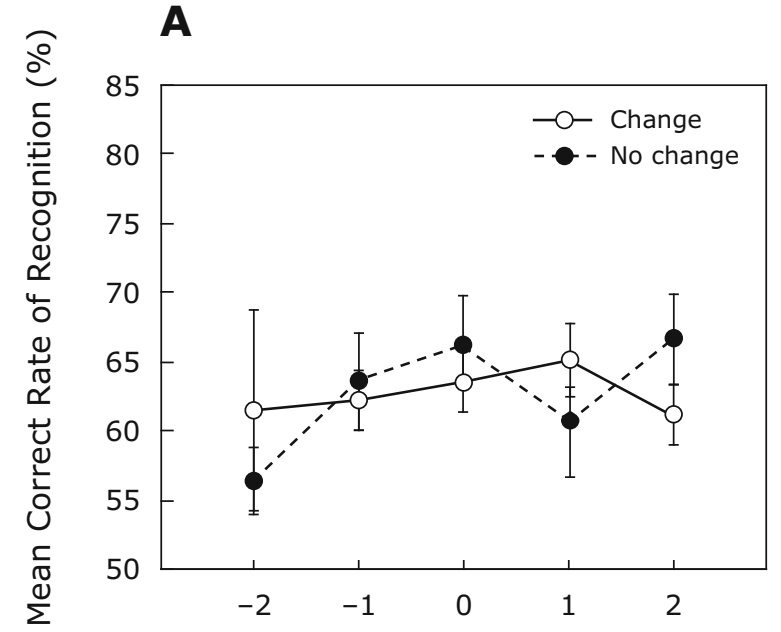

Temporal Position Relative to the Visual Change
B

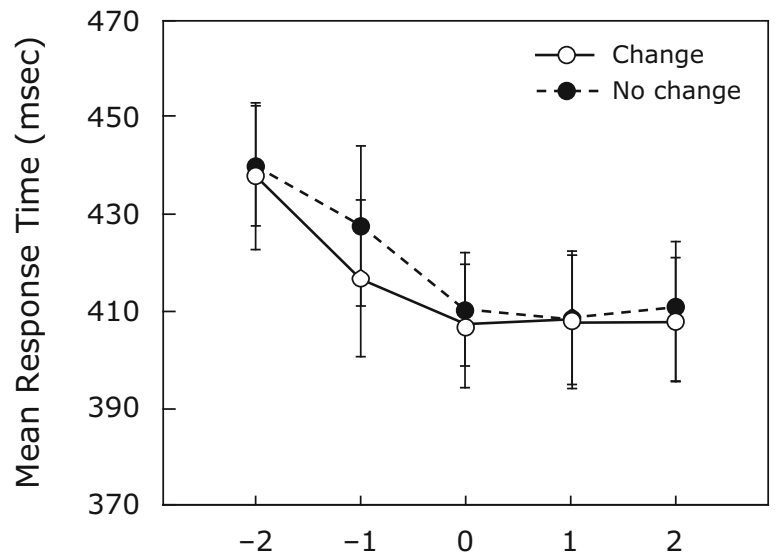

Temporal Position Relative to the Visual Change

Figure 3. (A) Recognition memory accuracy as a function of the temporal position of a letter in the sequence relative to the timing of the visual change in Experiment 2, averaged over participants. (B) Response times for target detection in Experiment 2 as a function of the temporal position of the target in a sequence relative to the timing of the visual change, averaged over participants. Error bars denote the standard errors of the means.

visual change (i.e., letters at +1 and +2 timings in the change condition) was not significant. We think that these discrepancies between the present experiments and those reported in the attentional capture literature may be due to the differences in presentation rate. The presentation rate used in our experiments was about $3 \mathrm{~Hz}$. This is much slower than is usual in rapid serial visual presentation experiments. Moreover, the previous report suggested that attentional capture could be produced by an irrelevant distractor at any spatial location in the visual field. However, our present results for Experiments 1 and 2 suggested that the participants' attention might be captured by a distractor that appeared only if the location of the visual change and the letter were the same.

\section{EXPERIMENT 3}

The experiments so far have suggested that a sudden visual change enhances recognition memory for a letter that appears close to it in space and time. In particular, Experiment 2 revealed that a spatial separation of the visual change from co-occurring letters eliminated the boost in recognition memory for these letters observed in Experiment 1. In Experiment 3, we considered two hypotheses concerning spatial manipulations. The first holds that a strict spatial coincidence of two separate entities, a visual change and a letter, is required for memory enhancement to occur. The second maintains that spatial coincidence is a special case of object formation and that, if a letter and visual change are perceived in relation to the same comprehensive object, a visual change will still elicit enhancement of recognition memory for the letter despite a spatial separation of the two. To test these hypotheses, we established a grouped spatial relationship between the visual change and letters.

\section{Method}

Participants. Seven new participants, with normal or correctedto-normal vision, were recruited for Experiment 3.

Stimuli and Procedure. The two black disks were spaced apart $\left(3.75^{\circ}\right)$ as in the previous experiments. In Experiment 3, however, they were connected by smooth curves to form a single dumbbelllike object, as shown in Figure 4. The minimum width of the connector was $1.1^{\circ}$. The size increase occurred in one of the two disks. The letter sequence occurred on the contralateral side of the visual change. In other respects, this experiment was identical to the two preceding experiments.

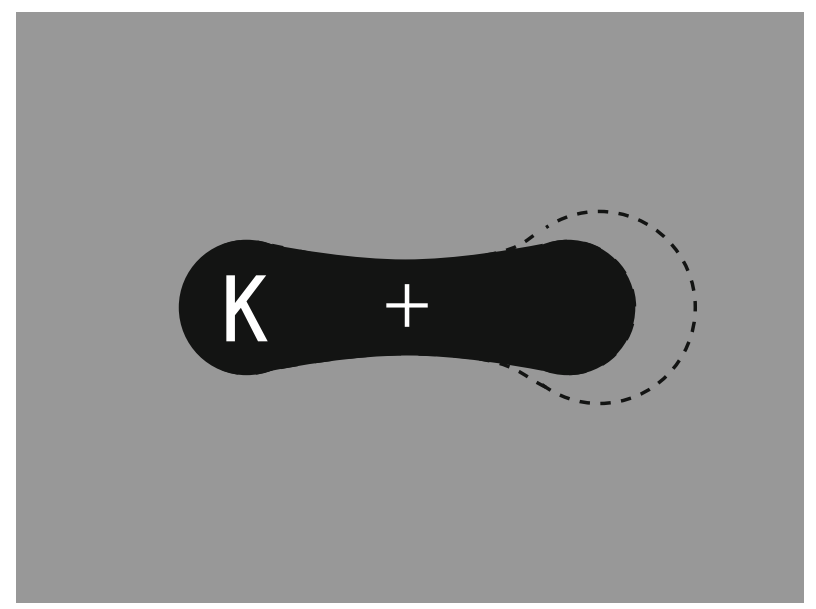

Figure 4. Dumbbell-shaped object used in Experiment 3. The standard (not expanded) disks were spaced equally apart, as in Experiment 2. In Experiments 3 and 4, the two disks were connected by smoothing curves. The minimum width of the connector was $1.1^{\circ}$. A dotted line depicts an outline of the object at the visual change in Experiment 3. At the disk expansion of the dumbbell-like object, one side of the disk, which was not the side with a letter sequence, expanded as large as in Experiments 1 and 2, remaining smoothly connected with another disk. 


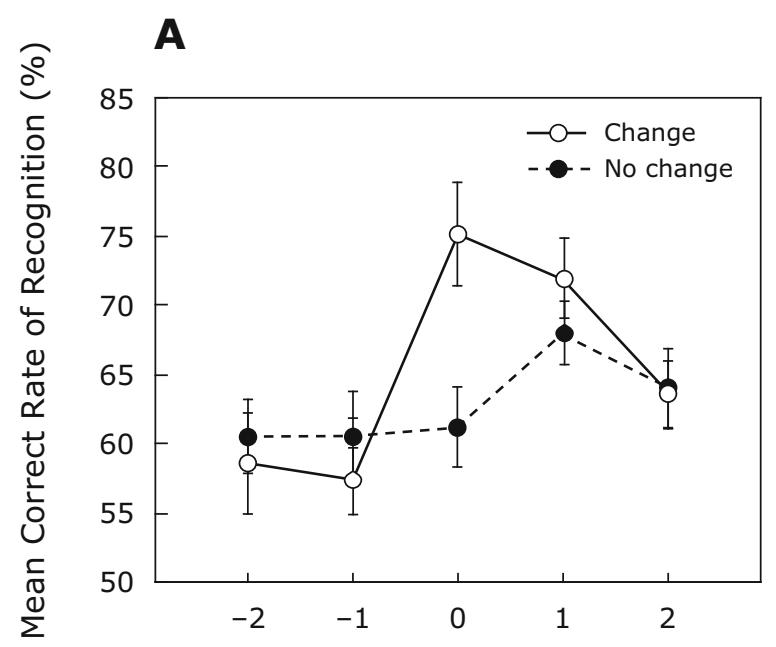

Temporal Position Relative to the Visual Change
B

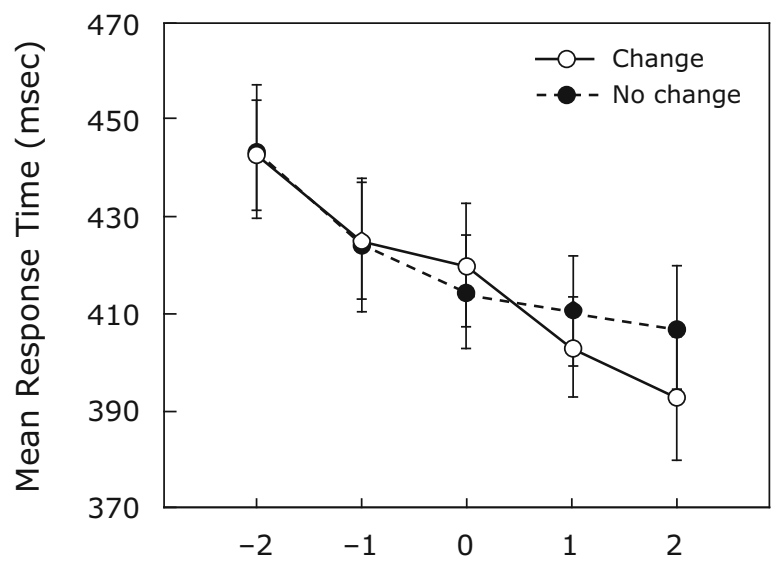

Temporal Position Relative to the Visual Change

Figure 5. (A) Recognition memory accuracy as a function of the temporal position of a letter in the sequence relative to the timing of the visual change in Experiment 3, averaged over participants. (B) Response times for target detection in Experiment 3 as a function of the target's temporal position in the sequence relative to the timing of the visual change, averaged over participants. Error bars denote the standard errors of the means.

\section{Results and Discussion}

Figure $5 \mathrm{~A}$ shows the results of the recognition memory task in Experiment 3. In the change condition, recognition accuracy peaked at zero, the case in which the visual change and the letter occurred simultaneously, resembling the results of Experiment 1. This enhancement occurred in spite of the spatial separation of the visual change and the letters. A repeated measures ANOVA revealed a significant main effect of relative temporal position $[F(4,24)=12.69, p<.01]$ and a significant interaction $[F(4,24)=3.02, p<.05]$. There was no significant main effect of change condition $[F(1,6)=4.69, p=.07]$. Post hoc Tukey's HSD tests indicated significant differences between letter recognition accuracy at zero in the change condition and that at the location of most other letters in the sequence. In addition, a significant difference was found between the recognition accuracy of the letter that appeared at the temporal position of the visual change ( \pm 0 relative timing of letter order) in both the change and no-change conditions. The differences between those at the other relative temporal positions $(-2,-1,+1,+2$ relative timing of letter order) in the change and no-change condition were not significant. As in the previous experiments, RTs in the detection task were shorter for targets presented later in the sequence [Figure 5B; significant main effect of relative temporal position, $F(4,24)=10.55, p<.01]$, but these did not differ between the change conditions $[F(1,6)=0.63$, $p=.46]$. There was no significant interaction $[F(4,24)=$ $1.45, p=.25]$.

The results of Experiment 3 showed that, even when a letter and a visual change were separated in space, the presence of a unified object enclosing them restored the enhancement of recognition memory for the letter that simultaneously appeared with the visual change. This result points to the possibility that memory enhancement by task-irrelevant visual changes is object based, rather than space based.

\section{EXPERIMENT 4}

The results of Experiment 3 indicated that memory enhancement by visual change is object based. To confirm the finding further, we examined, in Experiment 4, the case in which both space-based and object-based effects would occur; the disk enlargement occurred at the same side as the letter sequence of the dumbbell-like object.

\section{Method}

Participants. Seven new individuals participated in the experiment. All of them had normal or corrected-to-normal vision.

Stimuli and Procedure. The visual stimuli and procedure were the same as those in Experiment 3, except that the visual change (transient size increase of a black disk) occurred on the same side as the letter sequence. In all other respects, the two experiments were identical.

\section{Results and Discussion}

Figure 6A shows the results of the recognition memory task in Experiment 4. In the change condition, recognition memory accuracy showed a peak at the zero temporal position where the target letter and the visual change occurred simultaneously. A repeated measures ANOVA confirmed the significant interaction $[F(4,24)=5.56$, $p<.01]$. No other effects reached significance $[F(1,6)=$ $0.32, p=.59$, for change condition; $F(4,24)=2.17, p=$ .10 , for temporal position]. Post hoc Tukey's HSD tests revealed a significant difference between recognition accuracy at the zero temporal position ( \pm 0 relative timing of letter order) in the change and no-change conditions. The differences between those at other relative times in these conditions were not significant. In both conditions, detection times were shortened as the relative temporal 


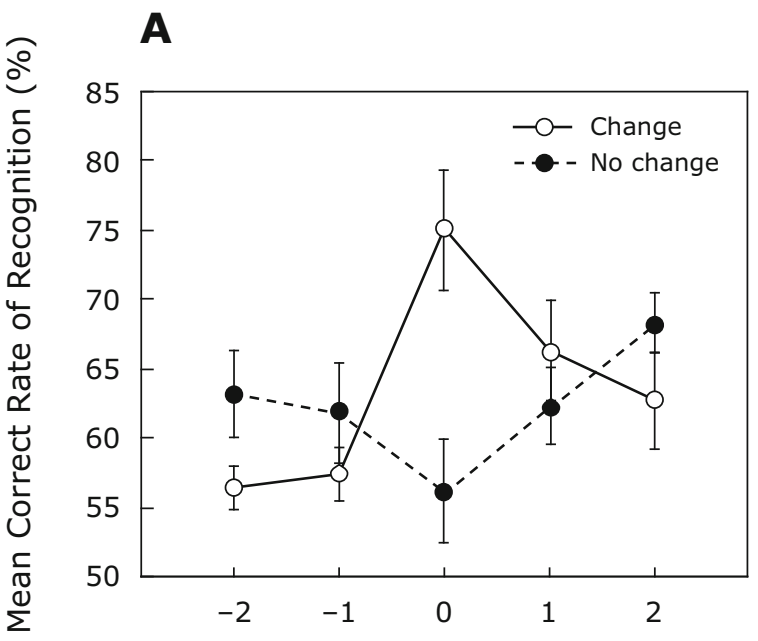

Temporal Position Relative to the Visual Change
B

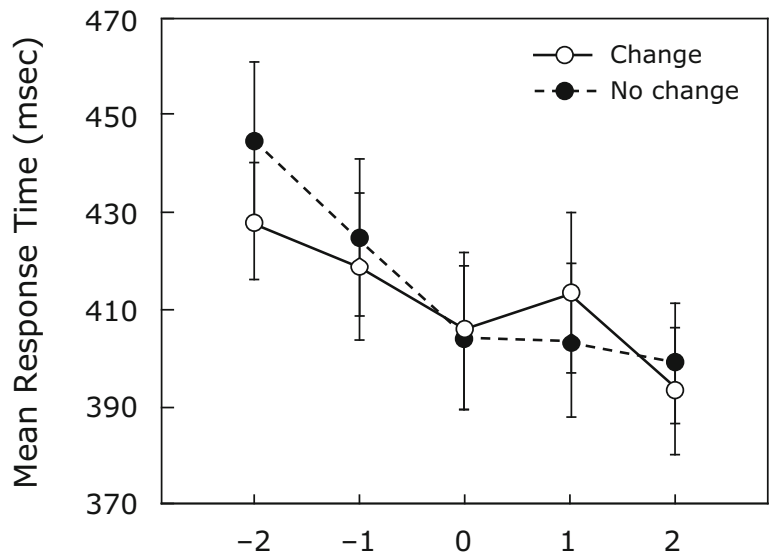

Temporal Position Relative to the Visual Change

Figure 6. (A) Recognition memory accuracy as a function of the temporal position of a letter in the sequence relative to the timing of the visual change in Experiment 4, averaged over participants. (B) Response times for target detection in Experiment 4 as a function of the target's temporal position in the sequence relative to the timing of the visual change, averaged over participants. Error bars denote the standard errors of the means.

position increased in the sequence [Figure 6B; significant main effect of relative temporal position, $F(4,24)=20.11$, $p<.01]$, but no significant effect was found for change condition $[F(1,6)=2.94, p=.14]$ and the interaction $[F(4,24)=1.92, p=.14]$. The results of Experiment 4 resembled those of Experiments 1 and 3 in showing that a visual change simultaneous with a letter enhanced recognition memory for the letter.

Upon finishing the four separate experiments, we conducted a between-experiments comparison of recognition accuracy in the change condition to summarize the effect of visual change on recognition memory enhancement. Figure 7 shows the effect of visual change on recognition memory (subtracting the percentage of memory accuracy in the no-change condition from the accuracy in the change condition) in all four experiments. In Experiments 1 and 4, the visual change occurred at the letter location. In Experiments 2 and 3, the change occurred at a different location from the letter, but in Experiment 3, the visual change and the letter occurred on the same object figure. In other words, the visual change and the letter appeared on the same object in Experiments 1, 3, and 4; however, in Experiment 2 , the change and the letter appeared on separate objects. The graph depicts that visual change tended to enhance recognition memory of a letter that simultaneously appeared with the change only when the change occurred on a part of the object, which integrated it with the letter. A between-participants mixed ANOVA (experiment [4] $\times$ change condition [2] $\times$ temporal position [5]) confirmed a significant main effect of temporal position $[F(4,24)=9.36, p<.01]$ and a significant interaction of temporal position and change condition $[F(12,72)=8.52$, $p<.01]$; a marginal significance was found for the main effect of change condition $[F(1,6)=4.09, p=.051]$ and for the three-factor interaction $[F(12,72)=1.78, p=.06]$. The main effect of experiment did not reach significance
$[F(3,18)=0.06, p=.98]$. Post hoc Ryan's tests showed that memory enhancement was significantly $(p<.05)$ larger for the zero temporal position in the change condition in Experiments 1, 3, and 4 than in Experiment 2. The results of the between-experiments comparison confirmed that the recognition memory of a letter was enhanced by a sudden visual change, providing that the change was presented at the same time and on the same object as the letter.
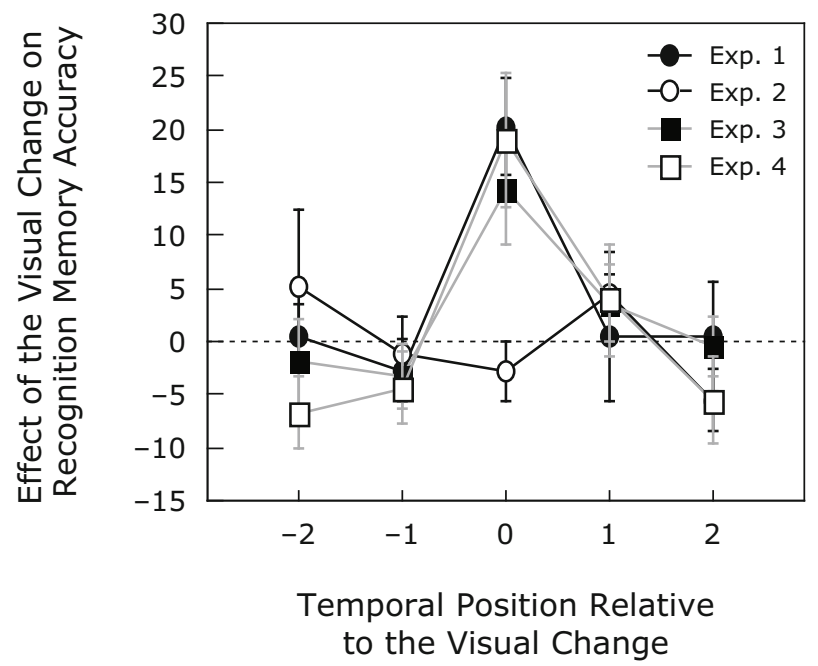

Figure 7. Effect of the visual change on recognition memory accuracy as a function of temporal position of a letter in the sequence relative to the timing of the visual change, averaged over participants, for Experiments 1, 2, 3, and 4. The enhancement effect of the visual change on memory accuracy was quantified by subtracting the percentage data of basic (no-change condition) memory accuracy from the change condition's percentage data. Error bars denote the standard errors of the means of the advantage of the visual change for memory accuracy. 


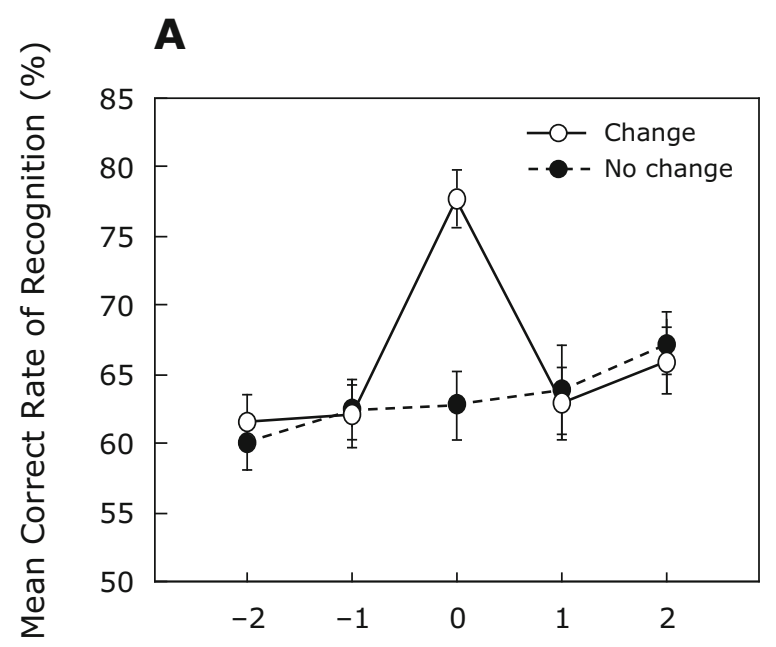

Temporal Position Relative to the Visual Change
B

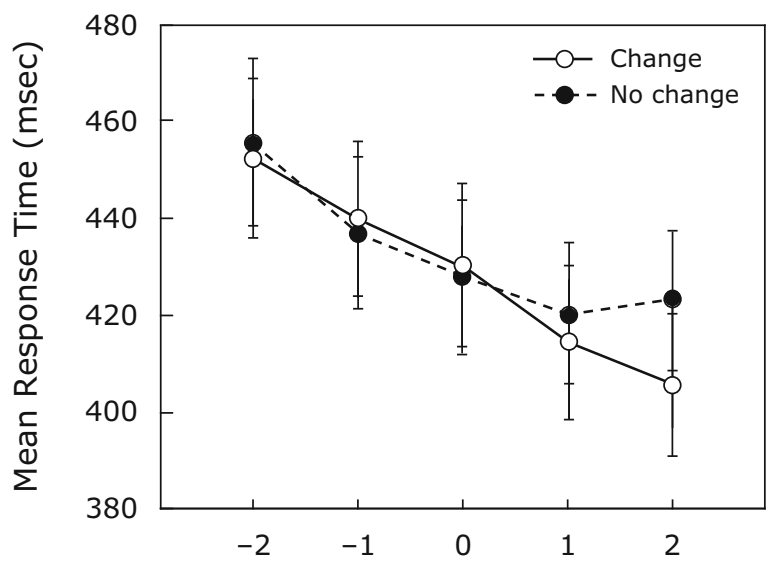

Temporal Position Relative to the Visual Change

Figure 8. (A) Response times for target detection as a function of the temporal position of a letter in the sequence relative to the timing of the visual change in Experiment 5, averaged over participants. (B) Response times for target detection in Experiment 5 as a function of the target's temporal position in the sequence relative to the timing of the visual change, averaged over participants. Error bars denote the standard errors of the means.

In addition, we informally asked the participants questions about their awareness of disk expansion in each experiment. The questions were, "Did you notice the visual change in every trial?" and "Did you think a letter that appeared with the visual change was more easy or difficult to remember (or detect) than other letters?" For the first question, all the participants reported that although they were aware that the disk expanded on some trials, they did not notice the change on most trials. The participants also reported that they thoroughly attended to the letter sequence and attended less to the visual change. For the second question, the participants reported that they did not think that the visual change had any effect on their task performance. These reports did not differ among participants between experiments. In other words, the participants were equally less attentive to and aware of the visual change in the four experiments.

\section{EXPERIMENT 5}

The results of the previous experiments indicated that the task-irrelevant visual changes enhanced recognition memory for a simultaneously presented letter in an object-based manner. However, in the experiments so far, the visual change occurred on half of the trials. Whether the temporal visual change would take place on a given trial was unpredictable, and it was still possible for the participants to adopt (intentionally or unintentionally) the strategy of selectively memorizing an item that appeared at the same time as the visual change. It has been shown that an abrupt change directs attention to a region in space even when it is counterpredictive of an item location (see, e.g., Jonides, 1981), indicating that the process is highly automatic. In Experiment 5, in order to confirm that the visual change in our tasks acted as an exogenous cue, we made the visual change counterpredictive of the timing of the target letter.

\section{Method}

Participants. Seven new individuals with normal or correctedto-normal vision participated in the experiment.

Stimuli and Procedure. The visual stimuli and procedure were the same as those in Experiment 1, except that the visual change occurred infrequently in simultaneous timing with the target letter to be asked about. In Experiment 1, all five temporal positions of targets around the time of the visual change were tested for recognition with equal probability (i.e., 20\%). In this experiment, the target letter presented at the visual change was tested on only $4 \%$ of the trials (i.e., the probability that the other letters would appear at $-2,-1,+1,+2$ temporal position was $24 \%$ each). More specifically, 36 trials were repeated for \pm 0 temporal positions, and 216 trials were repeated for each of the other four temporal positions, and then 900 change trials and 900 no-change trials were conducted. In addition, catch trials were incorporated; 30 trials for the peripheral six temporal positions were randomly inserted to eliminate the possible use of memorization for just the five letters at the temporal positions of interest around the visual change (360 trials). Thus, in total, 2,160 trials were performed.

\section{Results and Discussion}

Figure 8A depicts the results of the recognition memory task in Experiment 5. In the change condition, recognition memory accuracy showed a peak at the zero temporal position, at which the target letter and the visual change occurred simultaneously. A repeated measures ANOVA confirmed significant main effects for change condition $[F(1,6)=15.91, p<.01]$, for temporal position $[F(4,24)=$ $12.76, p<.01]$, and for the interaction $[F(4,24)=15.18$, $p<.01]$. Post hoc tests showed a significant difference between the change and no-change conditions only at the zero point. In both these conditions, RTs for the detection task shortened as the relative temporal position (timing of the letter in the sequence) became later [Figure 8B; 
$F(4,24)=61.14, p<.01]$. Differing from the previous experiments, a significant main effect of change condition $[F(1,6)=14.07, p<.01]$ and a significant interaction $[F(4,24)=4.66, p<.01]$ were found; the reason(s) for these effects are unclear.

The results of Experiment 5 resembled those of Experiment 1 in demonstrating that a task-irrelevant visual change that was simultaneous with a letter enhanced recognition memory for the letter. Despite the fact that the visual change was counterpredictive of the timing of the target letter, the memory improvement persisted in Experiment 5. Thus, it appears that the transient task-irrelevant visual change (i.e., the sudden disk enlargement) acted as an exogenous temporal cue for the task of memorizing serially presented letters and that the memory enhancement occurred rather automatically for the letter that appeared with the cue.

\section{GENERAL DISCUSSION}

In the present study, we sought to determine whether memory for serially presented items would be enhanced by task-irrelevant visual changes in an object-based manner. The experiments varied in terms of the background disks, the grouping of these with the connection, and their spatial relation with the letter sequence. The letter sequence occurred on the visual object (either the single disk or the dumbbell-like object) in Experiments 1, 3, 4, and 5, whereas it was on the other object in Experiment 2. From the observer's point of view, the relevance of the visual change might also differ among the experiments, where it occurred in the attended location or as part of the attended object, except for Experiment 2. Thus, although the disk enlargement was a task-irrelevant visual change in principle, it was still possible that it was significant in terms of memory processes when it occurred within the focus of attention as an exogenous visual cue. By assuming that the item and visual cue were taken as parts of the same object, this effect can be explained. Furthermore, memory enhancement by the visual cue automatically occurred even when the cue was counterpredictive of the timing of the item to be asked about. The results indicate that recognition memory for a visual stimulus is automatically enhanced when it is coincident with an exogenous visual cue in time and object-based space.

Previous studies have been focused on the input order of multiple (simultaneous) objects in memory. In free recall tasks, it has long been known that accuracy is higher for initial (primacy) and final (recency) items in a seriesthat is, the classic serial position effect (Frensch, 1994; Gershberg \& Shimamura, 1994; Murdock, 1962; Posner \& Boies, 1971). A similar U-shaped accuracy function was observed in our study; in the no-change condition, accuracy was higher for targets in the initial and final serial positions than for those in the middle ones (data not shown). Previous research has reported that memory for multiple targets is limited by working memory capacity in recall tasks (Baddeley, 2003; Cowan, 2001; Kintsch et al., 1999; Miller, 1956). A study of working memory updating in serial-item perception suggests that forgetting order directly reflects input order (Wolfe, Reinecke, \& Brawn, 2006). In those studies, participants were instructed to remember multiple items that had equal importance. The participants had to remember each of these items in an indicated order, even though a number of items were designed to surpass memory capacity. As a result, if the items had equal importance, a priority of memory for the items was arranged in an indicated (or force-attended) order; the memory for the oldest attended item was successively washed out and overwritten by the latest attended item with highest priority. This earlier research indicates that the accuracy of recognition is highest for the most recent items in a series and decreases for items from earlier portions of the series. On top of the ordering effect on memory, the present results indicate that the priority in working memory is increased by a salient visual change, even when the visual change is task irrelevant or counterpredictive. Moreover, it should be noted that, in the present set of experiments, there was no spatial uncertainty as to item location; the participants fully focused on the item (letter) sequence. Therefore, the disk enlargement must have acted as an exogenous temporal cue (Coull, Frith, Büchel, \& Nobre, 2000; Griffin \& Nobre, 2005). The main finding of the present study is that an exogenous temporal cue influences recognition memory in an object-based way. If attended information attains a stronger representation than does unattended information (e.g., Desimone \& Duncan, 1995; Yeshurun \& Carrasco, 1998, 1999), the former should endure longer time in memory. Thus, the present results may be regarded as a manifestation of the effect of a background stimulus on exogenous temporal attention.

How does an exogenous cue cause memory enhancement? One possibility is that a sudden oddball change (e.g., in size, color, and so on) in a visual object produces a subjective change in perceptual time (New \& Scholl, 2009; Ohyama \& Watanabe, 2007; Tse, Intriligator, Rivest, \& Cavanagh, 2004). Tse et al. reported that people faced with a salient event perceived time to pass more slowly. Recognition memory might be enhanced due to the perceptual dilation of item duration. However, a recent study of spatial properties of this subjective time dilation suggested that an oddball visual change expands the perceived duration of items in the entire visual field (New \& Scholl, 2009). In contrast, in the present experiments, recognition accuracy was affected only if the item was presented on a visual object that underwent a salient visual change. It seems, therefore, that improvement of recognition memory and subjective time dilation are based on at least partially different mechanisms, although it is possible that subjective time dilation might contribute to the enhancement of recognition memory.

An alternative explanation is suggested by the research of Vogel, Woodman, and Luck (2005). These researchers examined the time course of perception and memory for an array of items in response to a visual cue. The cue was an arrow pointing to the left or right side of a visual field and appeared simultaneously with the items. They found that the cue did not affect perception of the items but that it did affect memory for it. They suggested that a simultaneous cue-plus-item configuration means that the 
perception of the items was processed in parallel with the encoding of the cue at the same time; consequently, the effect of the cue (i.e., attentional shift) not only can have an impact on item perception, but also can influence postperceptual processes, such as memory for the items. This rationale can be applied to our paradigm if we assume that the visual change in our study functioned as a cue. Then, the lesser effect of visual change found in the detection task, which putatively measured attention/perception, would make sense. We discovered the effects of a visual cue on recognition of serially presented items, and this finding is consistent with this interpretation, since a visual cue that is presented simultaneously with an item should affect memory for the item but may not have an influence on the perception of it. In addition, the results of Experiment 3 add the qualification that a visual cue affects recognition memory for an item only if the cue can be taken to be a part of the same object with the item. This finding is in accordance with an earlier finding that bottom-up perceptual grouping cues can bias the entry of items into visual working memory (e.g., Woodman, Vecera, \& Luck, 2003).

Object-based memory enhancement could also be explained as an attention-driven effect during a perceptual process. Object-based cuing effects have been extensively studied in sensory (or attentional) processes (Behrmann et al., 1998; Egly et al., 1994; Moore et al., 1998; Shomstein \& Behrmann, 2008; Watson \& Kramer, 1999). These studies showed that when an item appeared on a part of a previously cued object, item detection became faster than detection of an item on another, uncued object, known as the object-based attentional advantage. Different from the previous studies, however, item location was always predictable in the present study. Therefore, the detection task in our study was easier than the target detection task used in the previous studies, and consequently, the objectbased effect of the exogenous cue might not appear in the RTs in our study; so there is still a possibility that the memory enhancement effect reflects the process of object-based attention.

Part of the explanation must involve object-based processing and its role in recognition memory, which has figured prominently in research (Fink, Dolan, Halligan, Marshall, \& Frith, 1997; Pei, Pettet, \& Norcia, 2002; Schumacher, List, Robertson, \& D'Esposito, 2001; Shomstein \& Yantis, 2002; Treisman, 1996). A prominent idea is that attention integrates the features of visual targets to form object percepts (Treisman, 1996). Selective attention brings together into one object a number of stimulus features within an array. In our experiments, participants focused on the target letter sequence. It occurred on the visual object that would change (a disk in Experiment 1, a dumbbell in Experiments 3 and 4) or not change (Experiment 2). Recognition memory performance for target letters might have been enhanced presumably because the integration of the target sequence and the visual object (disk or dumbbell) occurred routinely in the presence of focused attention. It would be interesting if the same pattern of results could be ob- tained when attention was averted to somewhere else in the visual field.

Does our finding of object-based memory enhancement have connections with memory phenomena in everyday life? The present study employed a recognition task in which the participants chose one out of two alternatives. However, in everyday life, a correct answer is not so frequently presented. To gain insight, we conducted a preliminary experiment in which participants were required to report as many of the letters presented in a sequence (i.e., recall task) as possible. The results showed that the letter appeared with the transient disk enlargement and tended to be recalled more often. Although the experiment was laborious and, therefore, an amendment is required for its implementation, the recall task would provide more information regarding whether memory capacity changes with an exogenous temporal cue and, if such a capacity is fixed, which item is excluded in exchange for the cued item.

In conclusion, the results of the present study suggest that if a salient visual event on an object happens to occur at close temporal proximity to a to-be-remembered item, our memory system assigns high priority to the item. However, it seems that the item must be registered as belonging to the same object. When people watch a peculiar changing scene, they can remember the details of this scene better than those of other scenes, but only to the extent that these details converge to form an object and an event. Further investigations using the present experimental paradigm will reveal the nature of the visual changes (e.g., spatial and temporal predictability related to temporal attention, the extent of object-based effects, and so on) underlying recognition memory enhancement by visual events.

\section{AUTHOR NOTE}

This study was supported by the Japan Society for the Promotion of Science to J.O.; by a Grant-in-Aid for Scientific Research from the Japanese Ministry of Education, Culture, Sports, Science, and Technology to K.W.; by the CASIO Science Promotion Foundation to K.W.; and by the Japan Science and Technology Agency to K.W. Correspondence should be addressed to J. Ohyama, Research Center for Advanced Science and Technology, University of Tokyo, 4-6-1, Komaba, Meguro-ku, Tokyo 153-8904, Japan (e-mail: jo@fennel.rcast.u-tokyo.ac.jp).

\section{REFERENCES}

BADDELEY, A. (2003). Working memory: Looking back and looking forward. Nature Reviews Neuroscience, 4, 829-839.

BAYLIS, G. C., \& Driver, J. (1992). Visual parsing and response competition: The effect of grouping factors. Perception \& Psychophysics, 51, 145-162.

Behrmann, M., Zemel, R. S., \& Mozer, M. C. (1998). Object-based attention and occlusion: Evidence from normal participants and a computational model. Journal of Experimental Psychology: Human Perception \& Performance, 24, 1011-1036.

Bundesen, C. (1990). A theory of visual attention. Psychological Review, 97, 523-547.

Bundesen, C., \& HaRms, L. (1999). Single-letter recognition as a function of exposure duration. Psychological Research, 62, 275-299.

Coull, J. T., Frith, C. D., Büchel, C., \& Nobre, A. C. (2000). Orienting attention in time: Behavioral and neuroanatomical distinction between exogenous and endogenous shifts. Neuropsychologia, 38, 808-819.

Cowan, N. (2001). The magical number 4 in short-term memory: A 
reconsideration of mental storage capacity. Behavioral \& Brain Sciences, 24, 87-114.

Desimone, R., \& Duncan, J. (1995). Neural mechanisms of selective visual attention. Annual Review of Neurosciences, 18, 193-222.

DunCAN, J., \& HumphreYs, G. (1989). Visual search and stimulus similarity. Psychological Review, 96, 433-458.

Egly, R., Driver, J., \& RAFAL, R. D. (1994). Shifting visual attention between objects and locations: Evidence from normal and parietal lesion subjects. Journal of Experimental Psychology: General, 123, 161-177.

Fine, M. S., \& Minnery, B. S. (2009). Visual salience affects performance in a working memory task. Journal of Neuroscience, 29, 80168021.

Fink, G. R., Dolan, R. J., Halligan, P. W., Marshall, J. C., \& Frith, C. D. (1997). Space-based and object-based visual attention: Shared and specific neural domains. Brain, 120, 2013-2028.

Folk, C. L., Leber, A. B., \& Egeth, H. E. (2002). Made you blink! Contingent attentional capture produces a spatial blink. Perception \& Psychophysics, 64, 741-753.

FRENSCH, P. A. (1994). Composition during serial-learning: A serial position effect. Journal of Experimental Psychology: Learning, Memory, \& Cognition, 20, 423-442.

Gershberg, F. B., \& Shimamura, A. P. (1994). Serial position effects in implicit and explicit tests of memory. Journal of Experimental Psychology: Learning, Memory, \& Cognition, 20, 1370-1378.

Griffin, I. C., \& Nobre, A. C. (2005). Temporal orienting of attention. In L. Itti, G. Rees, \& J. K. Tsotsos (Eds.), Neurobiology of attention (pp. 257-263). Amsterdam: Elsevier.

JACKSON, M. C., \& RAYMOND, J. E. (2008). Familiarity enhances visual working memory for faces. Journal of Experimental Psychology: Human Perception \& Performance, 34, 556-568.

JaCKSON, M. C., WU, C. Y., Linden, D. E. J., \& RAYMOND, J. E. (2009). Enhanced visual short-term memory for angry faces. Journal of Experimental Psychology: Human Perception \& Performance, 35, 363374.

JONIDES, J. (1981). Voluntary versus automatic control over the mind's eye's movement. In J. [B.] Long \& A. [D.] Baddeley (Eds.), Attention and performance IX (pp. 187-203). Hillsdale, NJ: Erlbaum.

JoNIDES, J., \& YANTIS, S. (1988). Uniqueness of abrupt visual onset in capturing attention. Perception \& Psychophysics, 43, 346-354.

Kintsch, W., Patel, V. L., \& Ericsson, K. A. (1999). The role of longterm working memory in text comprehension. Psychologia, 42, 186198.

Kramer, A. F., \& Jacobson, A. (1991). Perceptual organization and focused attention: The role of objects and proximity in visual processing. Perception \& Psychophysics, 50, 267-284.

LaVIE, N., \& Driver, J. (1996). On the spatial extent of attention in object-based visual selection. Perception \& Psychophysics, 58, 12381251.

LuCK, S. J., \& Thomas, S. J. (1999). What variety of attention is automatically captured by peripheral cues? Perception \& Psychophysics, 61, 1424-1435.

Miller, G. A. (1956). The magical number 7, plus or minus 2: Some limits on our capacity for processing information. Psychological Review, 63, 81-97.

Moore, C. M., Yantis, S., \& Vaughan, B. (1998). Object-based visual selection: Evidence from perceptual completion. Psychological Science, 9, 104-110.

Müller, N. G., \& Kleinschmidt, A. (2003). Dynamic interaction of object- and space-based attention in retinotopic visual areas. Journal of Neuroscience, 23, 9812-9816.

MURDOCK, B. B. (1962). The serial position effect of free recall. Journal of Experimental Psychology, 64, 482-488.

New, J. J., \& Scholl, B. J. (2009). Subjective time dilation: Spatially local, object-based, or a global visual experience? Journal of Vision, 9(2, Art. 4), 1-11.
NIEMI, P., \& NäÄTÄNEN, R. (1981). Foreperiod and simple reaction time. Psychological Bulletin, 89, 133-162.

OhyAma, J., \& Watanabe, K. (2007). Unpredictable visual changes cause temporal memory averaging. Vision Research, 47, 2727-2731.

Pei, F., Pettet, M. W., \& Norcia, A. M. (2002). Neural correlates of object-based attention. Journal of Vision, 2(9, Art. 1), 588-596.

PosNer, M. I. (1980). Orienting of attention. Quarterly Journal of Experimental Psychology, 32, 3-25.

Posner, M. I., \& Boies, S. J. (1971). Components of attention. Psychological Review, 78, 391-408.

Potter, M. C., Staub, A., Rado, J., \& O'Connor, D. H. (2002). Recognition memory for briefly-presented pictures: The time course of rapid forgetting. Journal of Experimental Psychology: Human Perception \& Performance, 28, 1163-1175.

Prinzmetal, W., Presti, D. E., \& Posner, M. I. (1986). Does attention affect visual feature integration? Journal of Experimental Psychology: Human Perception \& Performance, 12, 361-369.

Schmidt, B. K., Vogel, E. K., Woodman, G. F., \& Luck, S. J. (2002). Voluntary and automatic attentional control of visual working memory. Perception \& Psychophysics, 64, 754-763.

Schumacher, E. H., List, A., Robertson, L. C., \& D'Esposito, M. (2001). Object- and space-based visual attention in the human brain. NeuroImage, 13, S356.

Shomstein, S., \& Behrmann, M. (2008). Object-based attention: Strength of object representation and attentional guidance. Perception \& Psychophysics, 70, 132-144.

Shomstein, S., \& Yantis, S. (2002). Object-based attention: Sensory modulation or priority setting? Perception \& Psychophysics, 64, 41-51.

Treisman, A. (1996). The binding problem. Current Opinion in Neurobiology, 6, 171-178.

Tse, P. U., Intriligator, J., Rivest, J., \& Cavanagh, P. (2004). Attention and the subjective expansion of time. Perception \& Psychophysics, 66, 1171-1189.

Vogel, E. K., Woodman, G. F., \& Luck, S. J. (2005). Pushing around the locus of selection: Evidence for the flexible-selection hypothesis. Journal of Cognitive Neuroscience, 17, 1907-1922.

Watson, S. E., \& Kramer, A. F. (1999). Object-based visual selective attention and perceptual organization. Perception \& Psychophysics, 61, 31-49.

Wolfe, J. M., Horowitz, T. S., \& Michod, K. O. (2007). Visual attention required for robust picture memory? Vision Research, 47, 955-964.

Wolfe, J. M., Reinecke, A., \& Brawn, P. (2006). Why don't we see changes? The role of attentional bottlenecks and limited visual memory. Visual Cognition, 14, 749-780.

Woodman, G. F., Vecera, S. P., \& LucK, S. J. (2003). Perceptual organization influences visual working memory. Psychonomic Bulletin \& Review, 10, 80-87.

Yantis, S. (1996). Attentional capture in vision. In A. F. Kramer, M. G. H. Coles, \& G. D. Logan (Eds.), Converging operations in the study of visual selective attention (pp. 45-76). Washington, DC: American Psychological Association.

Yeshurun, Y., \& CARRasco, M. (1998). Attention improves or impairs visual performance by enhancing spatial resolution. Nature, $\mathbf{3 9 5}$, $72-75$.

YeshuRUn, Y., \& CARRASCO, M. (1999). Spatial attention improves performance in spatial resolution tasks. Vision Research, 39, 293-305.

\section{NOTE}

1. In the interview after the experiment, all the participants reported that they had not noticed that the timing of the visual change had been fixed.

(Manuscript received September 4, 2009; revision accepted for publication June 9, 2010.) 